\title{
Früher tot durch psychische Belastung
}

\section{Zahlreiche Forschungsergebnisse der letzten Jahre deuten auf einen Zusam- menhang zwischen psychischer Belastung und einem erhöhten Risiko früh- zeitiger Sterblichkeit hin, vermittelt etwa über die Entstehung von kardiovas- kulären Krankheiten oder Krebserkrankungen.}

- Die meisten prospektiven Studien zu diesem Thema untersuchten eher kleine Stichproben. Daher mangelt es bislang an einer reliablen Schätzung des Risikofaktors und einer genauen Untersuchung des Kausalzusammenhangs verringert psychische Belastung die Lebenswartung oder tritt sie als Folge einer somatischen Erkrankung auf? Und wenn klinisch relevante Angst oder Depression ein Risiko darstellen, welche Aussage kann für subklinische Symptome getroffen werden?

Im Rahmen des Health Survey for England wurden von 1994 bis 2004 elf unabhängige Studien mit identischem Design in Privathaushalten durchgeführt. Insgesamt nahmen 85261 Menschen an dem computerbasierten Interview teil. Die Daten von 75936 Probanden wurden mit den Sterberegistern bis 2008 abgeglichen. Grundlage für die vorliegende Metaanalyse bildet der Rohdatensatz der 68222 Teilnehmer, die zu Studienbeginn älter als 35 Jahre waren und keine relevanten somatischen Vorerkrankung aufwiesen.

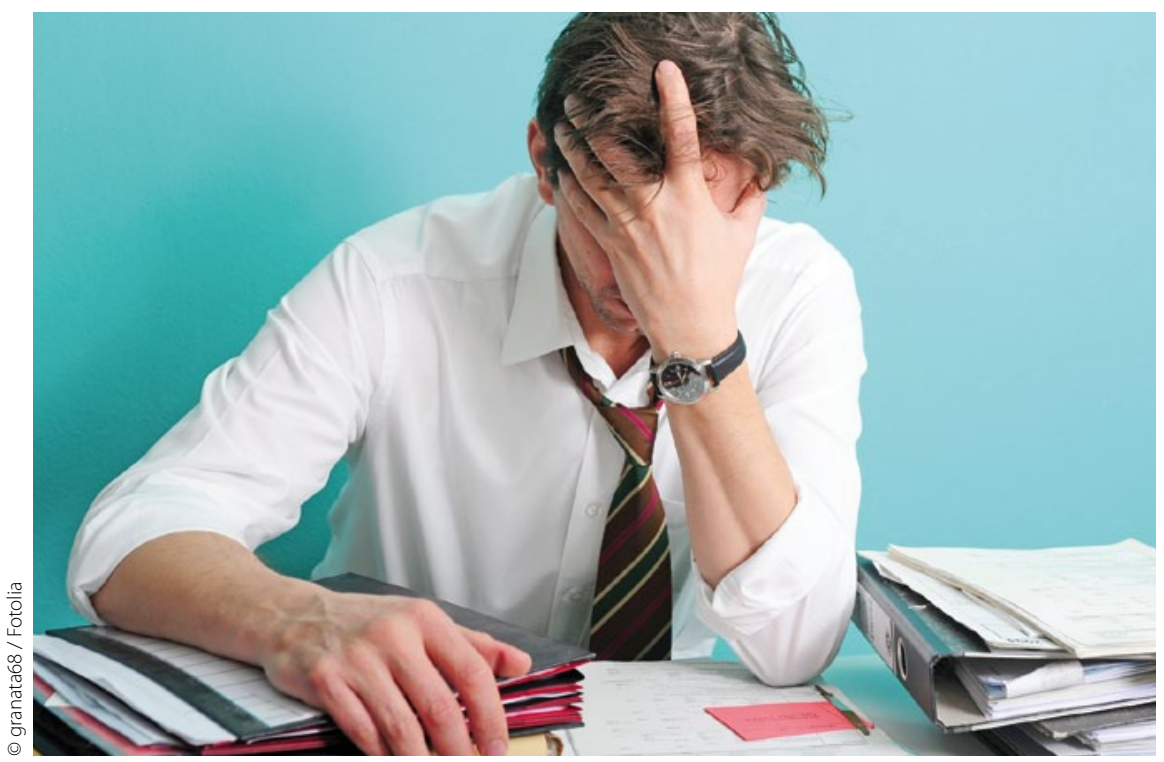

Mit dem Stress nimmt das koronare Risiko zu.
Die psychische Belastung wurde mit Hilfe des General Health Questionnaire (GHQ) erfasst, bei dem ab einem Gesamtwert von 4 von einer klinisch bedeutsamen Belastung auszugehen ist. Die aus den Sterbeurkunden gewonnenen Todesursachen wurden anhand von ICD-9 und 10 klassifiziert.

Als Maß für den Zusammenhang von GHQ-Score und Sterblichkeit wurden in einer Cox-Regressionsanalyse HazardRatios errechnet, wobei Teilnehmer mit Scores von 0 als Referenzgruppe verwendet und die Modelle an Alter, Geschlecht, Ausbildung, BMI, systolischen Blutdruck, Aktivitätsniveau, Raucherstatus, Alkoholkonsum und Vorliegen von Diabetes angepasst wurden.

Auf Basis der 8365 Todesfälle im Follow-up-Zeitraum (im Mittel 8,3 Jahre) konnte ein signifikanter Zusammenhang zwischen der psychischen Belastung der Teilnehmer zum Studienbeginn und der Sterbewahrscheinlichkeit gezeigt werden - unabhängig von der Schwere der Belastung und der Todesursache $(\mathrm{p}<0,001)$. Im Sinne einer Dosis-

Bei der vorgestellten Studie handelt es sich um die erste einzelfallbasierte Metaanalyse, die auf Grundlage einer großen bevölkerungsbasierten Stichprobe einen Zusammenhang zwischen psychischer Belastung und Sterberisiko zeigen konnte. Die genaue statistische Analyse unter Berücksichtigung zahlreicher relevanter Kontextvariablen erlaubt die Annahme, dass sich auch schon subklinisch ausgeprägte psychische Symptome kausal auf die Mortalität auswirken und dieser Effekt mit der Schwere der Belastung wächst. Lohnend für zukünftige Studien scheint die Frage, welche direkten (z. B. physiologischen) und indirekte (z. B. verhaltensmäßige) Prozesse hier zusammenwirken - und inwieweit der Effekt durch verschiedene Behandlungsansätze zu beeinflussen ist.

V. PAPE = 